\title{
Constraining magnetic fields and particle acceleration processes in galaxy clusters: a joint VLA/LOFAR view on Coma and SKA perspectives
}

\author{
Annalisa Bonafede ${ }^{* \dagger}$ \\ Hamburger Sternwarte, Universität Hamburg, Gojenbergsweg 112,21029 Hamburg (Germany). \\ E-mail: annalisa.bonafede@hs.uni-hamburg.de
}

I will present recent results on magnetic field and non thermal emission in galaxy clusters using VLA and LOFAR data. In particular, I will focus on the Coma cluster. I will discuss how the magnetic field estimates - obtained through Faraday Rotation Measure studies - and the new LOFAR observations will be able to constrain the particle acceleration processes in the radio halo and in the radio relic. I will then show how SKA already in its phase 1 will be able to improve the magnetic field estimates in a Coma-like cluster through a denser RM grid.

EXTRA-RADSUR2015 (*)

20-23 October 2015

Bologna, Italy

(*) This conference has been organized with the support of the Ministry of Foreign Affairs and International Cooperation, Directorate General for the Country Promotion (Bilateral Grant Agreement ZA14GR02 - Mapping the Universe on the Pathway to SKA)

* Speaker.

${ }^{\dagger}$ A footnote may follow. 


\section{Introduction}

Galaxy clusters host the largest-scale magnetic fields known to date (e.g. Feretti et al. 2012, and references therein). They could originate from an initial seed - amplified during the process of structure formation - or from magnetic fields injected by AGN or galactic outflows (e.g. Dolag et al. 2008; Donnert et al. 2009). The presence of magnetic fields in galaxy clusters can be probed by diffuse radio sources associated with clusters, and by Faraday rotation measures (RM) of embedded and background polarised sources (see e.g. Govoni \& Feretti 2004 for a review).

A fraction of galaxy clusters hosts diffuse radio emission on Mpc-scale, whose origin is still debated (e.g. Brunetti \& Jones 2014, and references therein). The emission originates from the IntraCluster Medium (ICM), and comes in a variety of shapes and morphologies. It has been classified into two different types of sources: radio halos and radio relics. All these sources have a steep spectrum $\left(\alpha>1\right.$, the spectrum is defined as $\left.S(v) \propto v^{-\alpha}\right)$, and are found in massive $\left(M \sim 10^{15} M_{\odot}\right)$ merging systems ${ }^{1}$. Mergers between massive clusters dissipate a huge quantity of gravitational energy in the ICM (up to $10^{64} \mathrm{ergs}$ ), and the big challenge of any theory is to channel part of this energy into the (re)acceleration of Cosmic Ray electrons and amplification of the magnetic field.

Radio halos could be produced by turbulent (re)acceleration of a population of mildly relativistic particles, while radio relics could result from (re)acceleration by shocks. We refer the reader to the reviews by Brüggen et al. (2011); Feretti et al. (2012); Brunetti \& Jones (2014) for the details. Although theoretical models can explain most $f$ the observed properties of halos and relics, they are still debated, and a complete understanding of the particle acceleration processes is still missing. Since the radio emission is due to the synchrotron process, one needs both constraints on the magnetic field and on the radio spectrum in order to constrain the energy spectrum of the emitting particles, and hence the (re)acceleration process.

Unfortunately, these sources have a weak surface brightness around $1 \mathrm{GHz}\left(\sim 1 \mu \mathrm{Jy} / \operatorname{arcsec}^{2}\right)$ and obtaining information on their spectra has been a challenge since their first discovery. In addition, the ICM magnetic field is unconstrained in most cases, because of the scarce number of polarised sources that present instruments can detect through the cluster.

The Coma cluster is so far the only case in which constraints on the magnetic field and on the radio spectrum of the radio halo and relic are available. Together with the wealth of gamma-ray, X-ray, optical and millimetric data, radio observations permit us to achieve a good understanding of the physical phenomena that are taking place in the ICM. Hence, the Coma cluster represents the prototype of the studies that all-sky radio surveys will be able perform on large samples of objects.

In this proceeding, I will illustrate the present constraints to the magnetic field in the Coma cluster (Sec. 2), and to the spectra of the radio halo and relic (Sec. 3). In Sec. 3 I will also show preliminary results from LOFAR (the LOw Frequency ARray) observations, and in Sec. 5 I will show the constraints to the magnetic field in a Coma-like cluster that will be obtained by the Square Kilometer Array (SKA).

\footnotetext{
${ }^{1}$ but see also Bonafede et al. (2014).
} 


\section{Magnetic field in the Coma cluster}

In the last decade, crucial improvements in understanding the magnetic field in galaxy clusters have been achieved, based partly on observations and partly on numerical simulations (e.g. Govoni et al. 2006, Dolag et al. 2008, Bonafede et al. 2011a, Vacca et al. 2012, Brüggen 2013). Much of what is known about the magnetic field in galaxy clusters comes from sensitive polarised observations. The observed polarisation angle $\phi_{o b s}$ of a synchrotron-emitting source observed in the background of a galaxy cluster is rotated with respect to the intrinsic polarisation angle by a quantity called Faraday Rotation Measure, defined as:

$$
R M=\int_{\text {cluster }} n_{e}(l) B_{/ /}(l) d l,
$$

where $n_{e}$ is the thermal gas density in the intra-cluster medium (ICM), and $B / /$ is the magnetic field component along the line-of-sight.

Bonafede et al. (2010) have used Faraday RM images of 7 sources located within/behind the Coma cluster to constrain the magnetic field profile. In Fig. 1 the RM images and the position of the sources throughout the cluster are shown. The magnetic field is assumed to scale with the thermal gas density, $n_{e}$ according to: $B(r) \propto B_{0} n_{e}^{\eta}$. The best agreement with observations is obtained for a magnetic field with a central magnetic field $B_{0} \sim 5 \mu \mathrm{G}, \eta=0.5$.

A radio relic is located in the SW of the cluster (e.g. Giovannini et al. 1993). If relics are connected to shock waves, one would expect to detect an enhancement of the RM in the radio relic region, as a consequence of the gas compression and magnetic field amplification by the shock. Bonafede et al. (2013) have observed 7 sources located in the SW region of the Coma cluster, in order to study the RM profile in that region and compare it with the profile derived in the cluster centre. In Fig. 1 the RM images of the sources in the SW region is shown. In that work, we have concluded that there is no "jump" in the RM for the sources detected through the radio relic, but a boost of the RM throughout the whole SW quadrant, and on both sides of the radio relic. The observed RM trend can be explained if the sources are lying onto a filament, where both gas density and the magnetic field are amplified. This filament could trace the accretion of gas, galaxies, and of the group NGC4872 towards the cluster centre.

In total, the magnetic field in the Coma cluster and in the relic region has been constrained using 14 radiogalaxies, and $\sim 150 \mathrm{~h}$ observing time at the Very Large Array. The sources that we have used are both cluster members and background objects, and the observed RM is assumed to be produced entirely the the ICM and by our Galaxy, whose contribution has been subtracted. Although we have performed several tests to verify our working hypothesis that the RM is due to the cluster, using only background sources would reduce the risk of local contamination (Guidetti et al. 2012). However, because of the low number density of polarised sources detectable with present instrument, using cluster members is still the only viable approach.

\section{Radio halo and relic}

The radio halo and the radio relic in the Coma cluster are probably the best studied. In the past years, people have used single-dish and interferometric observations to study the spectral 
properties of these sources from $\sim 150 \mathrm{MHz}$ up to $\sim 5 \mathrm{GHz}$ (Giovannini et al. 1993; Thierbach et al. 2003; Brown \& Rudnick 2011). As mentioned above, radio halos could be produced by turbulent motions, that develop in the ICM during cluster mergers. Turbulent motions could re-accelerate CRe up to $1-10 \mathrm{GeV}$, making them radio emitting over the cluster volume (re-acceleration models). The alternative models for radio halos (hadronic models) predict that they originate by hadronic collisions of CR protons with thermal protons, that through a decay chain form electron-positron pairs (Dolag \& Enßlin 2000; Enßlin et al. 2011). Using the magnetic field profile by Bonafede et al. (2010), the radio halo spectrum by Brown \& Rudnick (2011), and the upper limits from the Fermi satellite (Ackermann et al. 2014), it is possible to set constraints to the origin of the electrons that emit synchrotron radiation (Brunetti et al. 2012, 2013). In particular, these authors have concluded that the hadronic models for radio halos cannot reproduce the observed steepening at frequencies higher than $3 \mathrm{GHz}$.

The spectrum of the radio halo could only be obtained in the central region (within the inner 23', corresponding to $\sim 650 \mathrm{kpc}$ ) while most of the flux is emitted at larger radii (Brunetti et al. 2013). Hence, it is crucial to obtain deeper images of the radio halo in as many frequencies as possible, in order to constrain the spectrum in the outer parts of the halo.

The radio relic has been recently studied by Trasatti et al. (submitted, see also Trasatti et al, this conference) combining interferometric and single-dish observations. A single power-law spectrum seems to reproduce the radio emission, in agreement with the predictions from shock acceleration models (e.g. Brüggen et al. 2011, and references therein). The Mach number derived from the radio spectral index $(M \sim 3)$ is consistent with the results by Erler et al. (2015), who have used Planck data to constrain the pressure discontinuity imprinted by the shock in the ICM.

\section{New LOFAR observations}

In order to constrain the spectrum of the diffuse emission and its total extent, low frequency radio observations are needed. The Coma cluster has been observed by LOFAR within the Survey key-science project collaboration (Bonafede et al., in preparation). Observations were performed in the High Band (118-190 MHz) for a total time of 10 hours. Virgo A is at $\sim 17$ degrees from the Coma cluster, and its emission comes in and out of the beam, affecting the quality of the calibration. The multi-beam receivers of the telescope allowed us to observe in parallel to the Coma cluster a second source, 3C286, that is used as a calibrator. Specifically, we have used 3C286 to remove the average clock offset from the target field ${ }^{2}$, and to set the absolute flux scale. Once the clock offsets are removed from the target field, we have applied the amplitude gain solutions found for 3C286 to the Coma observations. The initial phase calibration has been done using a global sky model, that includes data from several radio surveys. Some cycles of phase self-calibration have been performed to refine the phase solutions. A more sophisticated approach, that takes the directionaldependent effects into account (the facet calibration, see van Weeren et al. 2016a) is underway. A preliminary analysis of the data shows promising features to understand the acceleration processes at work in the ICM. The radio halo has a total extent of $\sim 2 \mathrm{Mpc}$, while the relic is $\sim 800 \mathrm{kpc}$ long. In agreement with the results by Trasatti et al (submitted) at higher frequencies, there is no

\footnotetext{
${ }^{2}$ this is needed because the LOFAR Remote Stations are on separate clocks
} 

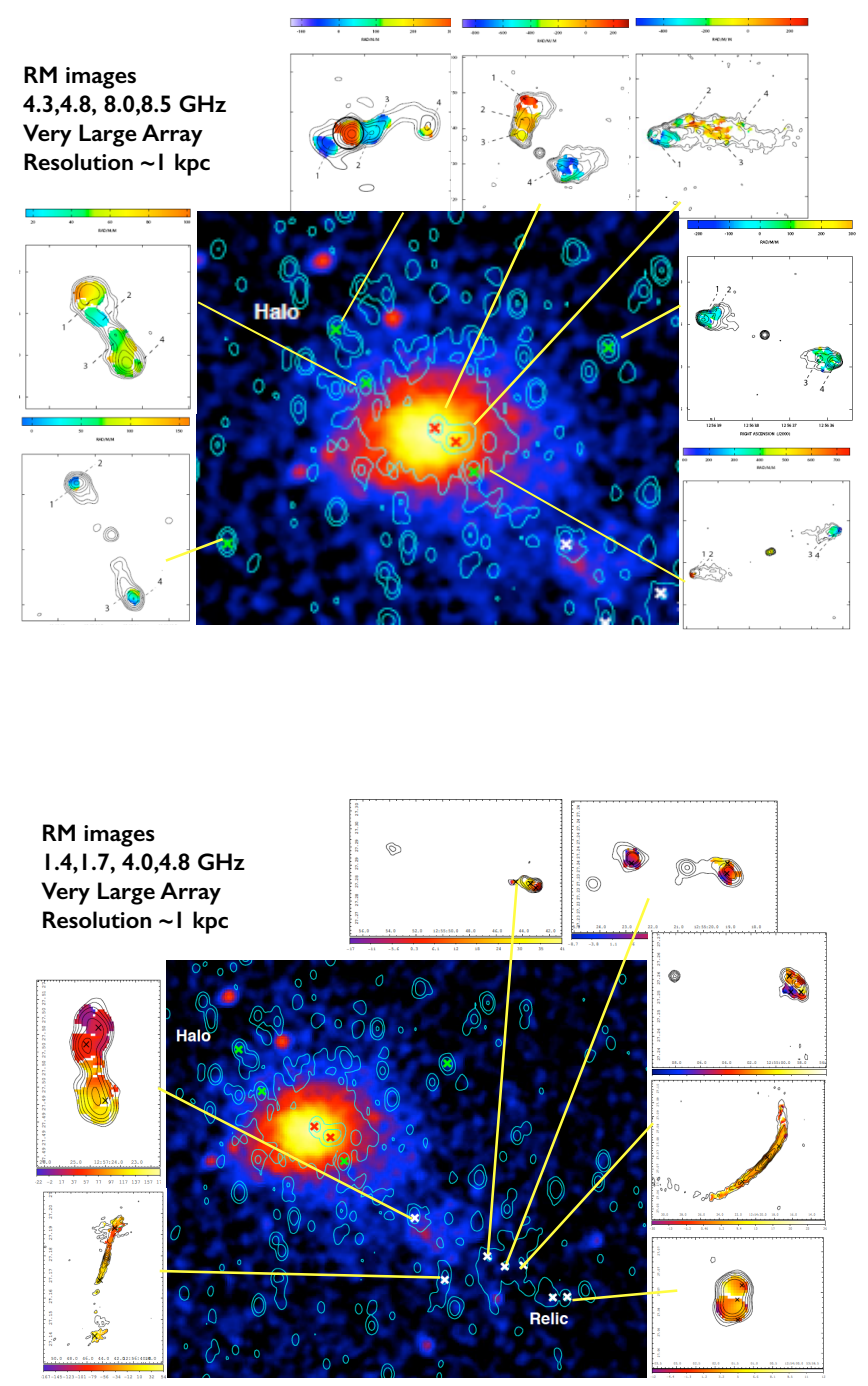

Figure 1: top: in the central panel color show X-ray emission from the Coma cluster as seen by ROSAT. Contours show the radio emission (Giovannini et al. 1993). The seven sources used to derive the RM are labelled with crosses. Their RM images are shown around the main panel(Bonafede et al. 2010). bottom: as in the top panel, but in the region of the radio relic (Bonafede et al. 2013).

evidence for a further extension of the relic towards North, as found by Brown \& Rudnick (2011). In addition, a low surface brightness bridge is connecting the relic and the halo, as already indicated by Giovannini et al. (1991); Brown \& Rudnick (2011), and diffuse emission is detected connecting the source NGC4789 to the radio relic on the SW side. Once the calibration and imaging will be finalised, LOFAR observations will permit to constrain the properties of these low-brightness radio emission regions. 


\section{SKA perspectives on the study of magnetic fields}

In the next months, LOFAR will complete the survey of the Northern sky (Tier 1 survey), and it will be possible to study the radio emission of already known radio halos and relics as well as discovering new ones (van Haarlem et al. 2013; van Weeren et al. 2016b). An equivalent survey to constrain the magnetic field properties in the ICM will only be possible with SKA. In order to predict the capabilities of the SKA in studying the magnetic fields on the large scales, we have produced mock RM observations of single radiogalaxies detected through the ICM. We have used an approach similar to the one described in Murgia et al. (2004) and Bonafede et al. (2010), that consists of modelling the gas distribution inside the galaxy cluster and the magnetic field in a separate 3-dimensional box. The magnetic field is then normalised within the core radius to a given value $B_{0}$, and is scaled according to:

$$
B \propto B_{0}\left(n_{e} / n_{0}\right)^{\eta}
$$

$n_{0}$ being the cluster central gas density as defined in the $\beta$-model. For further details we refer the reader to Bonafede et al. (2015) The SKA is expected to detect 315 polarised sources per square degree at at $1.4 \mathrm{GHz}$, with $2 \mu \mathrm{Jy}$ detection threshold and 2 arcsec resolution (Johnston-Hollitt et al. 2015). Assuming these number, we have computed the expected RM for every polarised background source, whose position is randomly extracted within the cluster field of view. This approach has the great advantage of using background objects only, and hence it minimises the possible local ICM contribution due to the interaction of cluster radio-galaxies with their environment. The magnetic field simulations are performed with the MiRo' code (see Bonafede et al. (2013) for further details).
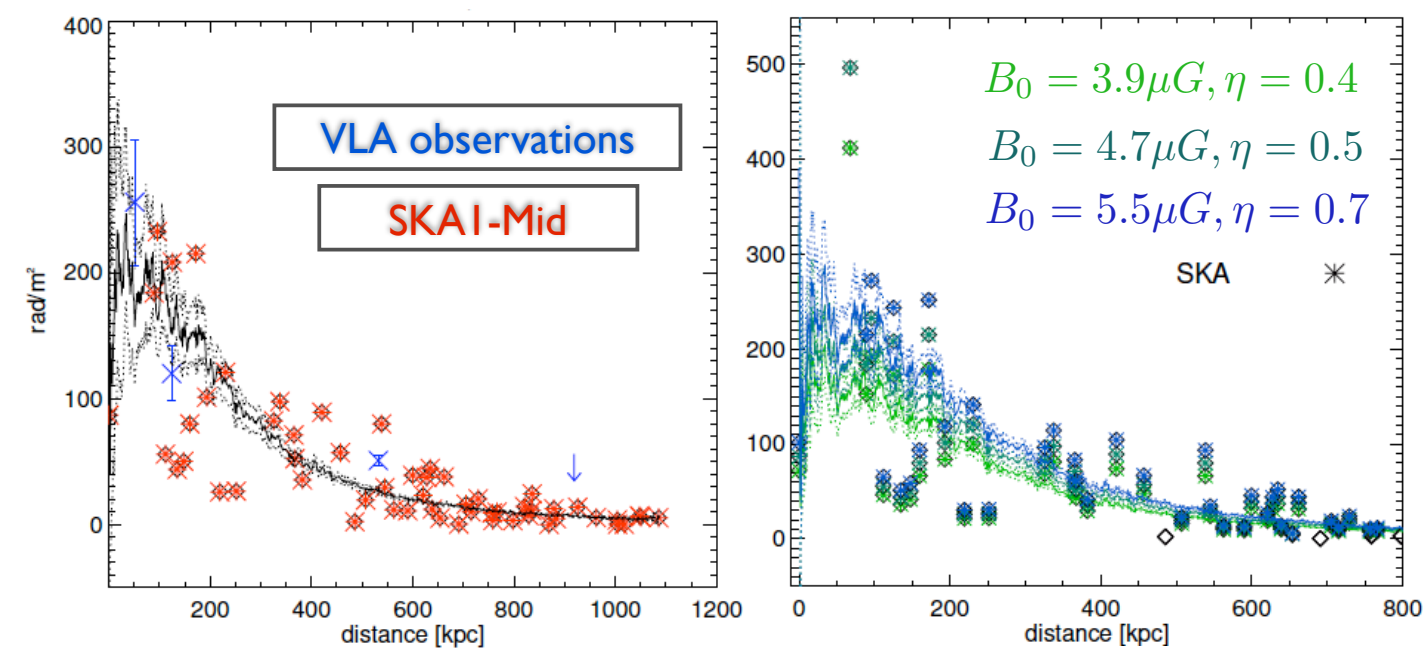

Figure 2: SKA1 predictions for the RM for Coma-like clusters. Left panel: Average profile of the RM (continuous line) and $5 \sigma$ dispersion (dotted lines) obtained for a Coma-like cluster. Diamonds represent the sources that the SKA1 will detect in the background of the cluster. Red asterisks mark the sources whose RM is in the range to be detected by the SKA1. Blue points refer to the observations presented in Bonafede et al. (2010). Right Panel: lines and symbols are like in the left panel, shown in different colours for three different models as indicated in the inset. Figure from Bonafede et al. (2015) 
To model the gas distribution, we have assumed a $\beta$-model profile Cavaliere $\&$ Fusco-Femiano (1976). Our $\beta$-model clusters are meant to illustrate the big step forward allowed by the SKA in studying the magnetic field properties of galaxy clusters, compared to what has been achieved today with pointed observations of individual radiogalaxies.

The magnetic field in the Coma cluster is among the best constrained, and as such, it is a good starting point to investigate the capabilities of the SKA compared to the present facilities.

We have considered a cluster gas density profile that follows the $\beta$-model Cavaliere \& FuscoFemiano (1976) derived for the Coma cluster by Briel et al. (2001). For the magnetic field simulation, we have adopted the parameters that give the best fit to the Coma cluster magnetic field profile. We have simulated the different values of $B_{0}$ and $\eta$ that give the best agreement with the observations at $68 \%$ confidence level Bonafede et al. (2010). The different values of $B_{0}$ and $\eta$ leave an imprint on the RM profile, as shown in Figure 2. In Fig. 2 the RM profile obtained with SKA1 observations is shown for a "Coma-like" cluster. The results by Bonafede et al. (2010), obtained with VLA pointed observations, are overplotted. The surveys planned with the SKA1 will allow one to detect the RM from $\sim 50$ sources in the background of the "Coma-like" clusters. Thanks to the SKA1 field-of-view and sensitivity, similar results for a single cluster will be achieved with a single pointing. In Fig. 2, we also show the RMs obtained by the SKA1 for the different values of $B_{0}$ and $\eta$ that fit the VLA observations within the $68 \%$ confidence level. The resolution in RM of the SKA1 will enable us, in principle, to distinguish among these models, although a more accurate evaluation of the errors needs to be done to definitively assess this point.

\section{Conclusions}

In this proceeding, I have shown the present constraints to magnetic field and particle acceleration processes in the Coma Cluster, discussing the improvements that will be possible $n$ the next years thanks to instruments such as LOFAR and SKA. Since these instruments will survey the whole sky, the studies that are today possible on single, well studied objects, will become possible for a large sample of clusters.

\section{References}

Ackermann, M., Ajello, M., Albert, A., et al. 2014, ApJ, 787, 18

Bonafede, A., Feretti, L., Murgia, M., et al. 2010, A \& A, 513, A30

Bonafede, A., Govoni, F., Feretti, L., et al. 2011a, A \& A, 530, A24+

Bonafede, A., Vazza, F., Brüggen, M., et al. 2013, MNRAS, 433, 3208

Bonafede, A., Intema, H. T., Brüggen, M., et al. 2014, MNRAS, 444, L44

Bonafede, A., Vazza, F., Brüggen, M., et al. 2015, Advancing Astrophysics with the Square Kilometre Array (AASKA14), 95

Briel, U. G., Henry, J. P., Lumb, D. H., et al. 2001, A \& A, 365, L60 
Brown, S., \& Rudnick, L. 2011, MNRAS, 412, 2

Brüggen, M. 2013, MNRAS, arXiv:1308.5230

Brüggen, M., Bykov, A., Ryu, D., \& Röttgering, H. 2011, Science \& Space Review, 138

Brunetti, G., Blasi, P., Reimer, O., et al. 2012, MNRAS, 426, 956

Brunetti, G., \& Jones, T. W. 2014, ArXiv e-prints, arXiv:1401.7519

Brunetti, G., Rudnick, L., Cassano, R., et al. 2013, A \& A, 558, A52

Cavaliere, A., \& Fusco-Femiano, R. 1976, A \& A, 49, 137

Dolag, K., Bykov, A. M., \& Diaferio, A. 2008, Space Science Reviews, 134, 311

Dolag, K., \& Enßlin, T. A. 2000, A \& A, 362, 151

Donnert, J., Dolag, K., Lesch, H., \& Müller, E. 2009, MNRAS, 392, 1008

Enßlin, T., Pfrommer, C., Miniati, F., \& Subramanian, K. 2011, A \& A, 527, A99

Erler, J., Basu, K., Trasatti, M., Klein, U., \& Bertoldi, F. 2015, MNRAS, 447, 2497

Feretti, L., Giovannini, G., Govoni, F., \& Murgia, M. 2012, AAR, arXiv:astro-ph/12051919

Giovannini, G., Feretti, L., \& Stanghellini, C. 1991, A \& A, 252, 528

Giovannini, G., Feretti, L., Venturi, T., Kim, K.-T., \& Kronberg, P. P. 1993, ApJ, 406, 399

Govoni, F., \& Feretti, L. 2004, International Journal of Modern Physics D, 13, 1549

Govoni, F., Murgia, M., Feretti, L., et al. 2006, A \& A, 460, 425

Guidetti, D., Laing, R. A., Croston, J. H., Bridle, A. H., \& Parma, P. 2012, MNRAS, 423, 1335

Johnston-Hollitt, M., Govoni, F., Beck, R., et al. 2015, Advancing Astrophysics with the Square Kilometre Array (AASKA14), 92

Murgia, M., Govoni, F., Feretti, L., et al. 2004, A \& A, 424, 429

Thierbach, M., Klein, U., \& Wielebinski, R. 2003, A \& A, 397, 53

Vacca, V., Murgia, M., Govoni, F., et al. 2012, A \& A, 540, A38

van Haarlem, M. P., Wise, M. W., Gunst, A. W., et al. 2013, A \& A, 556, A2

van Weeren, R. J., Williams, W. L., Hardcastle, M. J., et al. 2016a, ArXiv e-prints, arXiv:1601.05422

van Weeren, R. J., Brunetti, G., Brüggen, M., et al. 2016b, ArXiv e-prints, arXiv:1601.06029 